\section{EMBRYRIDDLE \\ Aeronautical University}

SCHOLARLY COMMONS

\section{International Journal of Aviation,} Aeronautics, and Aerospace

\title{
Innovative Dodecacopter Design - Bulgarian Knight
}

Svetoslav Zabunov

Space Research and Technology Institute -- Bulgarian Academy of Sciences, svetoslavzabunov@gmail.com

Garo Mardirossian

Space Research and Technology Institute -- Bulgarian Academy of Sciences, garo.mardirossian@gmail.com

Follow this and additional works at: https://commons.erau.edu/ijaaa

Part of the Aeronautical Vehicles Commons

\section{Scholarly Commons Citation}

Zabunov, S., \& Mardirossian, G. (2018). Innovative Dodecacopter Design - Bulgarian Knight. International Journal of Aviation, Aeronautics, and Aerospace, 5(4). https://doi.org/10.15394/ijaaa.2018.1293

This Special Purpose Document is brought to you for free and open access by the Journals at Scholarly Commons. It has been accepted for inclusion in International Journal of Aviation, Aeronautics, and Aerospace by an authorized administrator of Scholarly Commons. For more information, please contact commons@erau.edu. 


\section{Introduction}

Unmanned aerial vehicles (UAVs) are gaining-ever greater importance to industry, law enforcement, homeland security, and defence. They are employed in protecting borders, tracking down smugglers, and delivering vital commodities to places inaccessible by means of ground or marine transportation. Apart from that, UAVs are used for teaching purposes too. The rapid development of drones creates challenges to researchers. New designs are being invented with better capabilities and parameters of flight performance and other benefits.

The unique construction of the novel multi-copter model Bulgarian Knight, disclosed in the current article, defines the rotors mounting position to be under the airframe, offers optimal rotor area coverage, and employs 12 rotors instead of 4, 6, or 8 rotors as encountered on most small electrically powered, currently manufactured UAVs. See an image of the prototype of the proposed design in Figure 1.

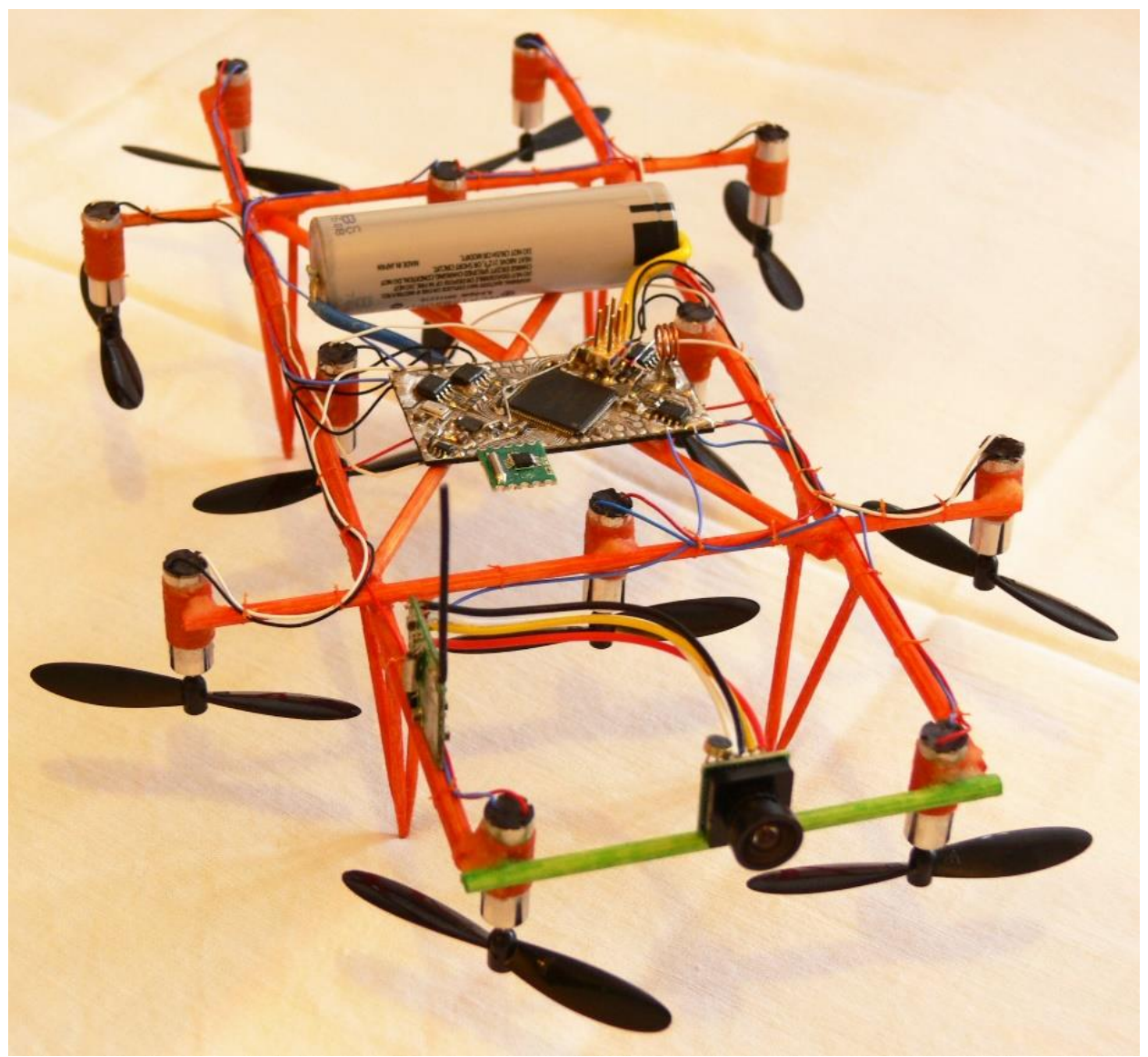

Figure 1 - Prototype of the twelve rotor multi-copter Bulgarian Knight. Dimensions: 280 x 185 x $40 \mathrm{~mm}$. 
Compared to current 12-rotor designs, Bulgarian Knight has unique rotor and airframe topology. Most, if not all, contemporary dodecacopters are based on the "star" topology (see Figure 2). Some of the commercially available units are:

- Gryphon Dynamics Dodeca (Figure 2b topology)

- OnyxStar Hydra-12 (Figure 2a topology)

- Carrier Hx12 Coaxial Dodecacopter Drone (Figure 2a topology)

Dodeca-multi-rotors with the design topology shown on Figure 2c could be spotted a few years ago, though even then these were very rare. The coaxial models (Figure 2a and $2 \mathrm{~b}$ ) have a pair of rotors mounted on each arm in a coaxial counter-rotating scheme. This approach minimizes airframe dimensions and complexity but increases disc loading and sacrifices lift efficiency (Maisel, Giulianetti, \& Dugan, 2000). Further, there is an increase in noise with this configuration (Block, 1986; Hanson, 1985).

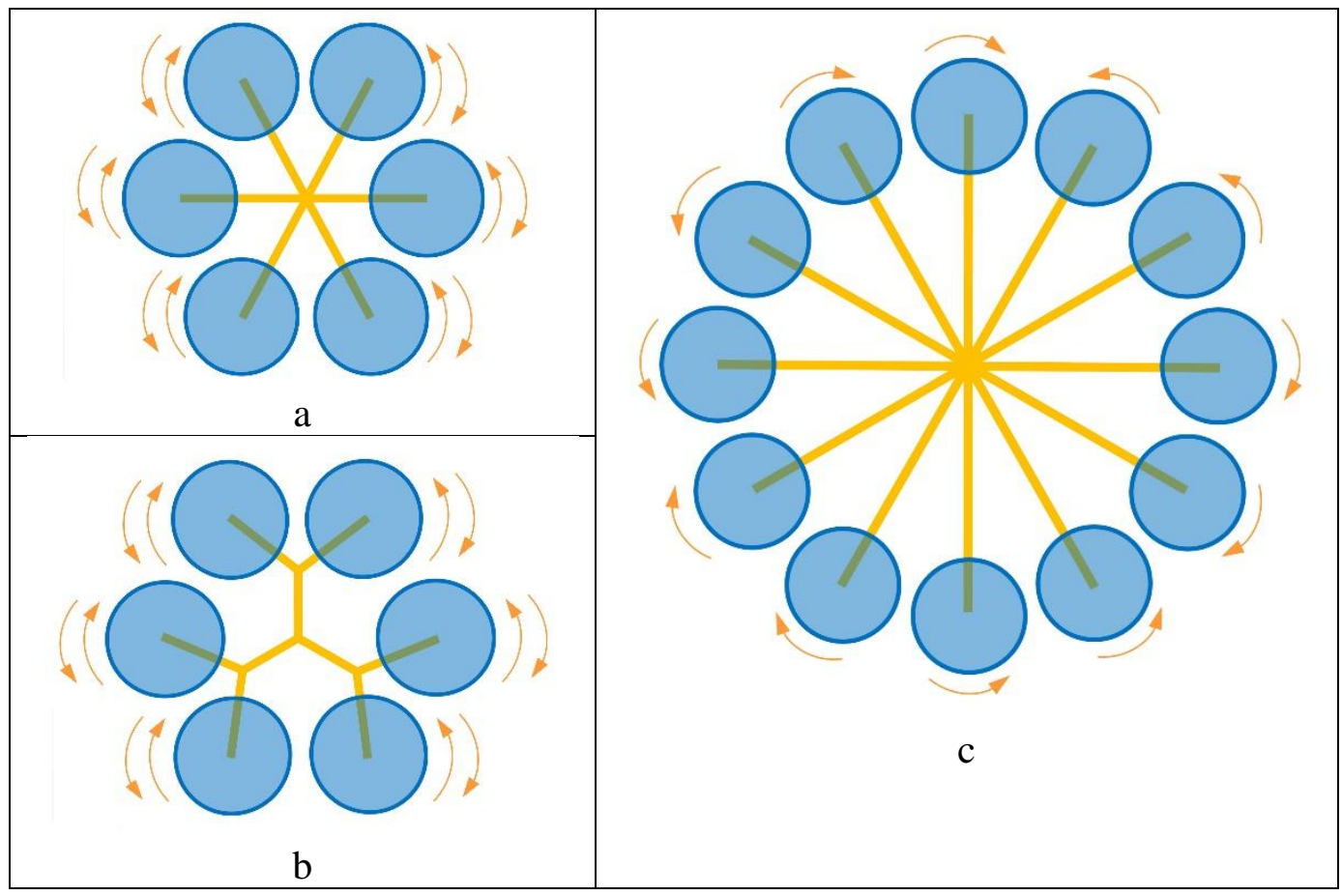

Figure 2 - Existing dodecacopter star-topology designs: coaxial dodecacopter with six arms (a); coaxial dodecacopter having three arms with "V" sections (b); full star dodecacopter with twelve arms (c). 


\section{Construction}

The general schematic of the multi-copter is shown in Figure 3. The construction of the aircraft is developed around three concepts:

1. Mounting the rotors under the fuselage.

2. Employing 12 rotors instead of 4,6 , or 8 rotors, as usually encountered on general purpose drones.

3. Implementing an optimal geometric coverage of the rotors.

There are several benefits gained from implementing these three concepts. First of all, the rotors are mounted under the fuselage in an off-body undermount manner as described in Yoon, Diaz, Boyd, Chan, and Theodore (2017) and Theys, Dimitriadis, Hendrick, and DeSchutter (2016). The distance between the propeller disc and the motor hub of 0.6 the rotor radius, while in Yoon et al. (2017) this measure is 0.25 of the rotor radius. The propellers are working in a pusher configuration. By having a significant distance between the propeller discs and the airframe, the propeller induced flow interference with the airframe/fuselage is diminished. By these means, three benefits are achieved:

- Improvement of the aerodynamic efficiency of lift due to lower interference of the rotor induced flow with the fuselage (Theys et al., 2016).

- Significantly less thrust oscillations are achieved. Otherwise, these would decrease the stability of the aircraft (Yoon et al., 2017).

- Decrease of the noise that is normally created by propeller wash exerting pulsating dynamic pressure on the airframe in the case of overmount rotors.

The authors have conducted an experiment using a laboratory setting to test the effect of pusher, instead of tractor propeller configuration. The test description and results are shown later in this document. 


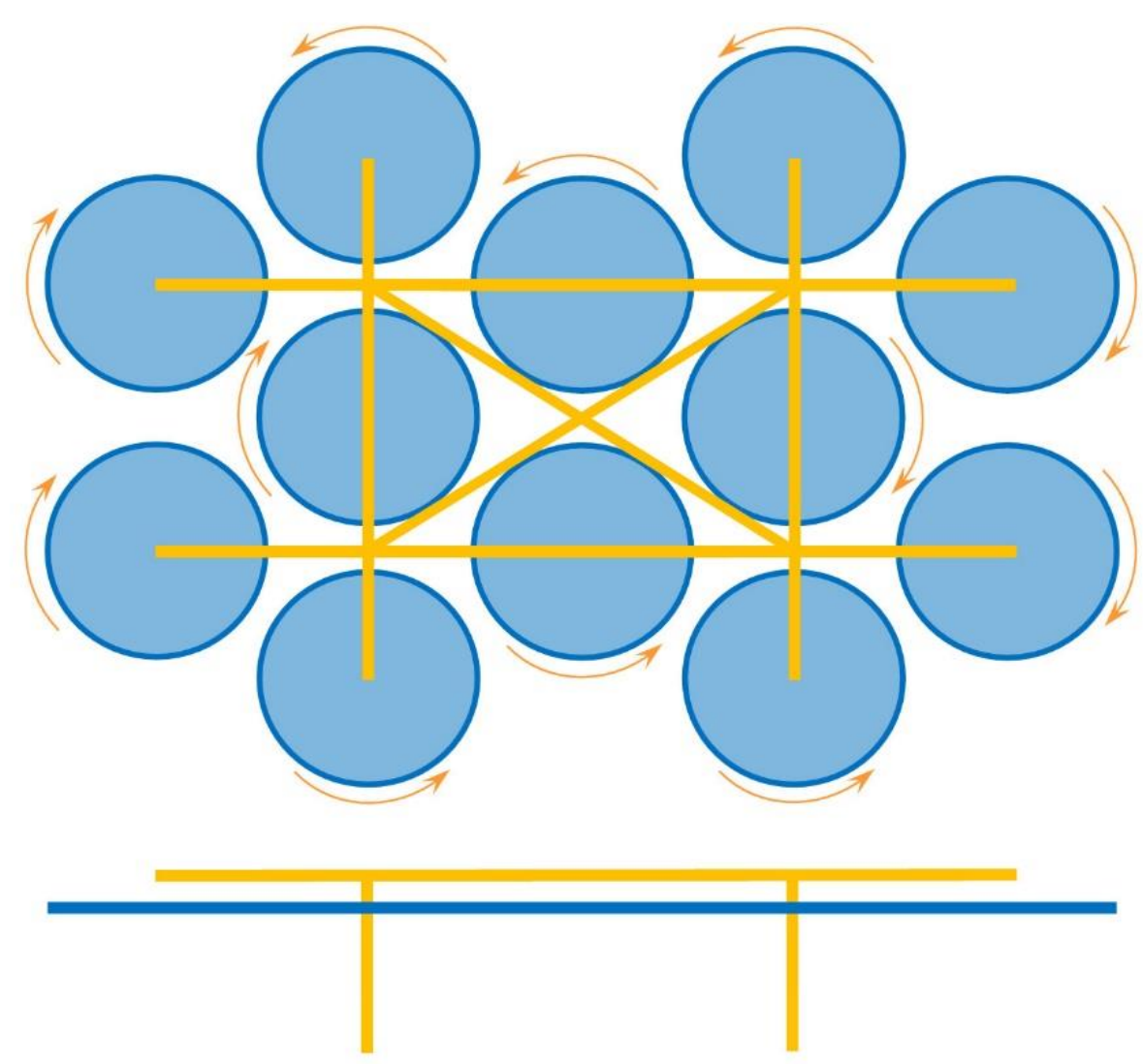

Figure 3 - General view of dodecacopter model Bulgarian Knight: Top View (above) and Side View (below).

The next idea in the proposed design is an increase in the number of rotors. A larger number of rotors has benefits as well as drawbacks. The most obvious shortcomings are increased complexity and, both larger and heavier airframe required to hold them. However, implementing the honeycomb scheme and increasing the number of rotors moderately - from the standard designs of 4 , 6 , and 8 rotors to 12 rotors - does not detrimentally raise the complexity of the multi-copter. The benefits from having more rotors are surpassing the disadvantages to a great extent. The advantages are as follows:

- Increased reliability. Failing of any of the rotors will not make the multi-rotor uncontrollable. The current design allows for simultaneous failure of up to four rotors.

- Lower noise and vibrations. The vibrations caused by the rotors in UAVs is a significant issue in the drone optimization domain. A number of authors have addressed this problem and sought to solve it 
through different means (Marichal, Rodriguez, \& Rivera, 2013; Radkowski \& Szulim, 2014; Verbeke \& Debruyne, 2016). By introducing a larger number of smaller rotors, the noise and vibrations frequency band will raise towards higher frequencies where vibrations filtration through mechanical dumping is much easier. Further, higher frequencies of noise will propagate to much shorter distances (Piercy $\&$ Embleton, 1977) and multi-copters with a large number of small propellers will be harder to intercept through acoustic signature.

- Increased safety. The damage a rotor could cause through impact on an object or a living being is proportional to its kinetic energy. When engaging a large number of small rotors, and because the contact is likely to occur with a single rotor, absorption of a smaller fraction of kinetic energy would result. The same is true when a propeller disintegrates - a smaller fraction of the total rotor kinetic energy of all rotors will be released through its debris, in this case 1/12 part of the total rotor energy.

The third novel approach is to implement an optimal geometric coverage of the 12 rotors, thus minimizing airframe dimensions and weight (see Figure 3 and Figure 4). This method guarantees minimal overall multi-copter dimensions and smallest possible airframe. The weight of the aircraft, too, is thus minimized. The densest circles packing in one plane is the hexagonal one also called honeycomb packing (Steinhaus, 1999). Its packing density is

$$
\eta_{h}=\frac{1}{6} \pi \sqrt{3} \approx 0.9069
$$

As mentioned earlier, in the "star" topology dodecacopters (Figure 2), each rotor is mounted to one end of an arm and all arms are arranged radially and connected in their other ends in the centre of the "star"-like airframe. A large gap is thus formed around the centre of the fuselage where no rotors are employed (see Figure 2). Hence, the "star" topology lacks the benefit of optimal rotor packing.

\section{Prototype development}

The authors of the current article are in the process of developing a prototype of the proposed design. The current model is shown in Figure 1, while a schematic with dimensions is presented in Figure 4. 


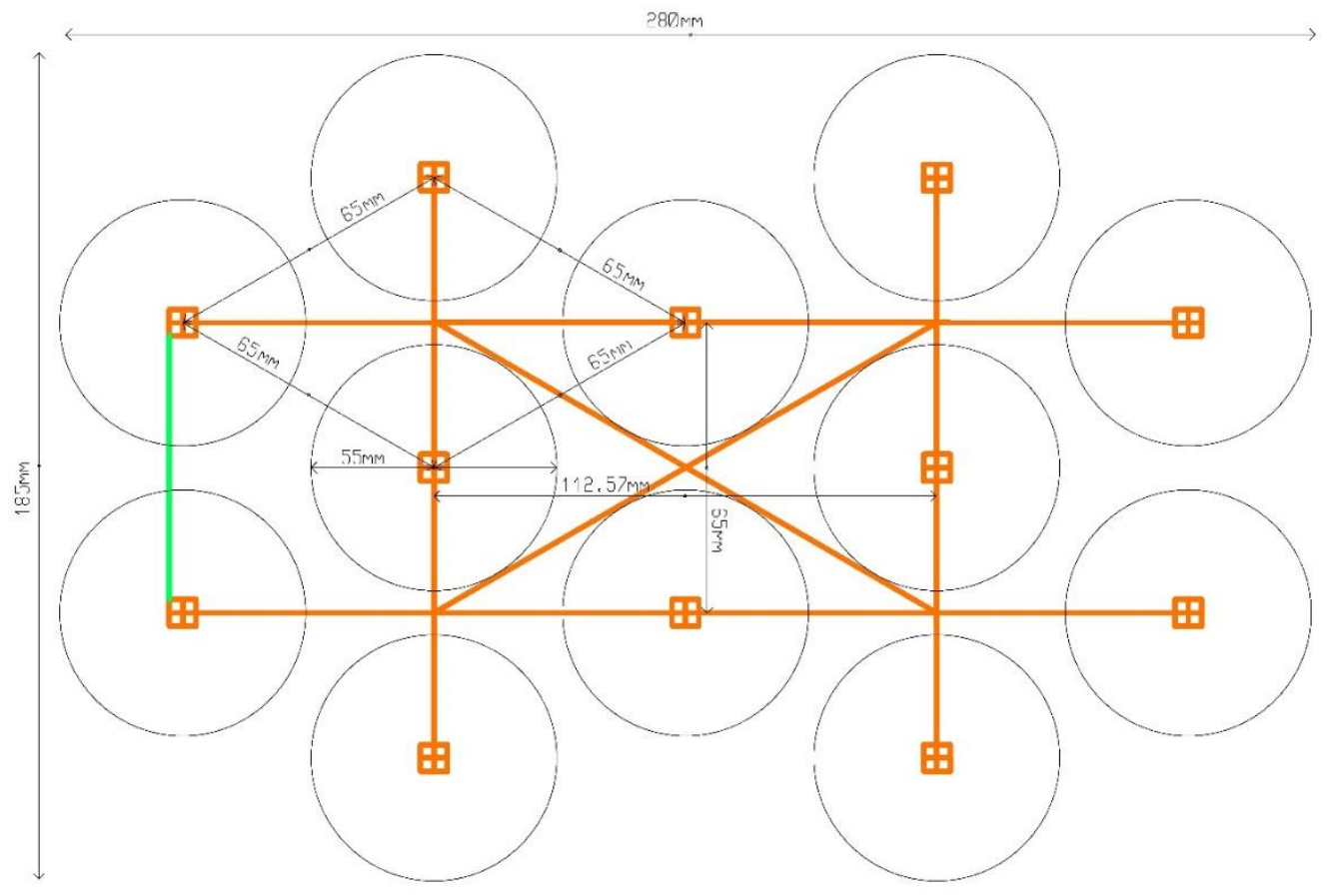

Figure 4 - Prototype design schematic with dimensions.

\begin{tabular}{llll}
\hline Part & Quantity & Single weight $[\mathrm{g}]$ & Total weight $[\mathrm{g}]$ \\
\hline Battery & 1 & 50 & 50 \\
Motor & 12 & 4 & 48 \\
Propeller & 12 & 0.5 & 6 \\
Autopilot with radio receiver & 1 & 6 & 6 \\
Camera & 1 & 3 & 3 \\
Video radio transmitter & 1 & 2 & 2 \\
Airframe & 1 & 6 & 6 \\
\hline Total weight & & & 121 \\
\hline
\end{tabular}

Table 1. Prototype parts with their weight

A micro drone prototype has been elaborated. The development is in an advanced stage and all parts have been mounted to the airframe. Table 1 summarizes the parts with their weight.

The used motors are brushed micro-motors of the $7 \mathrm{~mm} \times 20 \mathrm{~mm}, 0.9 \mathrm{~mm}$ shaft, type. The propellers are $55 \mathrm{~mm}$ in diameter, plastic, with clockwise and counter clockwise rotation. There is a standard NTSC video camera mounted with 120 degrees field of view and a $2.4 \mathrm{GHz}$ video transmitter. The autopilot is a development of the first author and has integrated inertial navigation system 
consisting of 3D MEMS gyroscope, 3D MEMS accelerometer, 3D digital compass and barometer. The autopilot also realizes motor speed controllers, and a VHF radio receiver. The total weight of the prototype has been measured and the measurement process photo is shown in Figure 5.

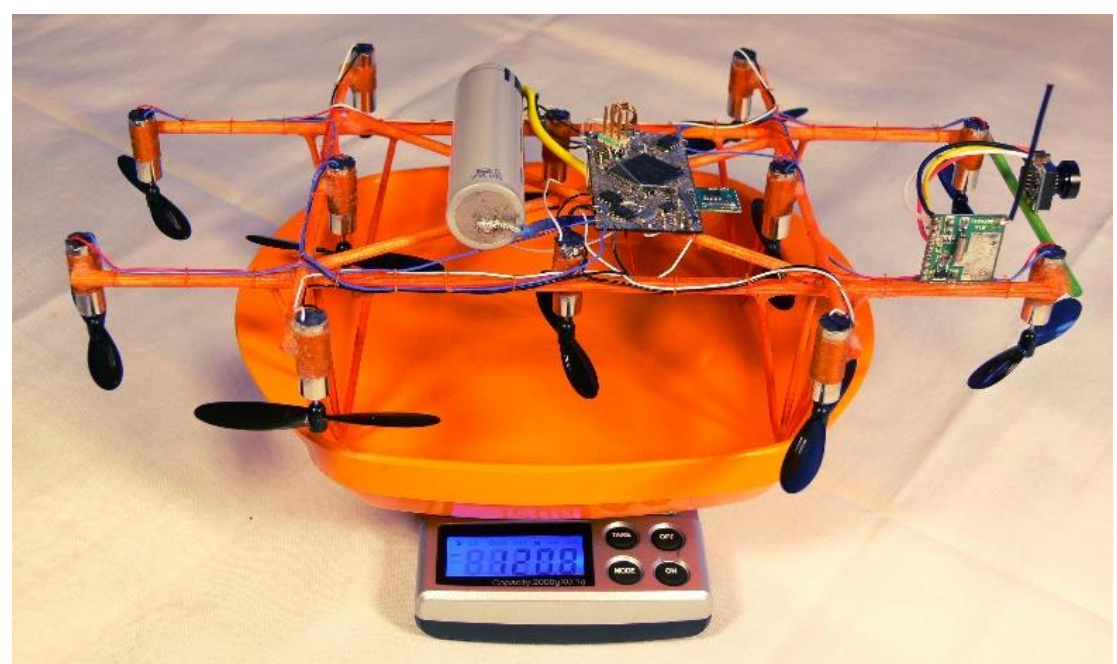

Figure 5 - Total weight of the multi-rotor prototype equals $120.8 \mathrm{~g}$.

Why have we developed a microdrone prototype instead of a larger aircraft? Microdrones are gaining increasing interest due to their low cost, being easy to carry, capable of negotiating windows and doors of buildings and flying indoors. Microdrones do not create high noise while flying and are thus hard to acoustically detect and track. They are also very hard to spot visually or though radar reflections. Microdrones could be manufactured and employed in larger quantities for the same financial budget. Finally, there are legislative measures in many countries now entering into effect that would require stringent regulations on larger drones (above $250 \mathrm{~g}$ FFA drone rules in the USA). For these reasons we have picked out this prototype dimensions for our experiments and testing.

The used battery is a Li-Ion single cell rechargeable battery of the Panasonic 18650 NCR BD type. This battery allows max discharge continuous current of $10 \mathrm{~A}$. Its rated capacity is $3 \mathrm{Ah}$ and its nominal voltage is $3.6 \mathrm{~V}$. The energy capacity of the battery is $10.50 \mathrm{Wh}$.

The landing gear/legs need to be placed at appropriate points on the airframe (Figure 3) in order not to get in the way of the rotor discs. The airframe has five such points available for mounting of the landing gear. Four landing legs are mounted on four of the cross points of the airframe's beams - the centre cross point of the fuselage is not employed. 


\section{Motor thrust scales}

For the purpose of testing a pusher versus tractor propeller approach, we have created a testing scale (Figure 6). The scale has three arms of equal length. The instrument is in equilibrium when the weighing dishes are empty (Figure 6 left). Because the prototype weighs $120.8 \mathrm{~g}$ and there are twelve rotors, each rotor is required to lift $10.07 \mathrm{~g}$ in order to sustain a hovering flight. In the experiment shown in Figure 6, a $10 \mathrm{~g}$ weight was used and was placed first in the right weighing dish (Figure 6 - middle). The propeller in this situation is of the tractor type. For generating $10 \mathrm{~g}$ static thrust the motor consumes $0.86 \mathrm{~A}$ at $1.9 \mathrm{~V}$, which equals $1.634 \mathrm{~W}$. When, on the same motor, a pusher propeller with the same dimensions and profile was mounted and the weight of $10 \mathrm{~g}$ was moved to the left weighing dish, the motor required $0.82 \mathrm{~A}$ at $1.7 \mathrm{~V}$ or $1.394 \mathrm{~W}$ to create the $10 \mathrm{~g}$ static thrust. This is a gain of $14.7 \%$ in power savings. When using a pusher propeller, the propeller induced flow (the propeller downwash) is not creating dynamic pressure on the scales arm it is mounted on. There is still interference of the induced flow with the motor arm, but it decreases rotor thrust significantly less. The saved power may be used to prolong the flight time and increase the aircraft's radius of operation.
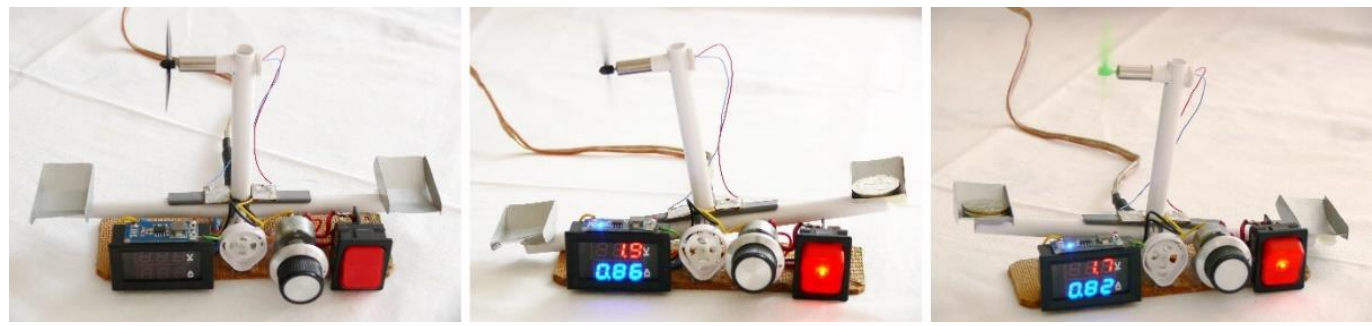

Figure 6 - Laboratory scale for propeller static thrust measurement. Left - scale in equilibrium; middle -measuring $10 \mathrm{~g}$ of static thrust in tractor propeller configuration; right - measuring $10 \mathrm{~g}$ of static thrust in pusher propeller configuration.

\section{Test setup}

A test setup was advised for the purpose of measuring the benefit of pusher undermount rotors instead of tractor overmount rotors. 


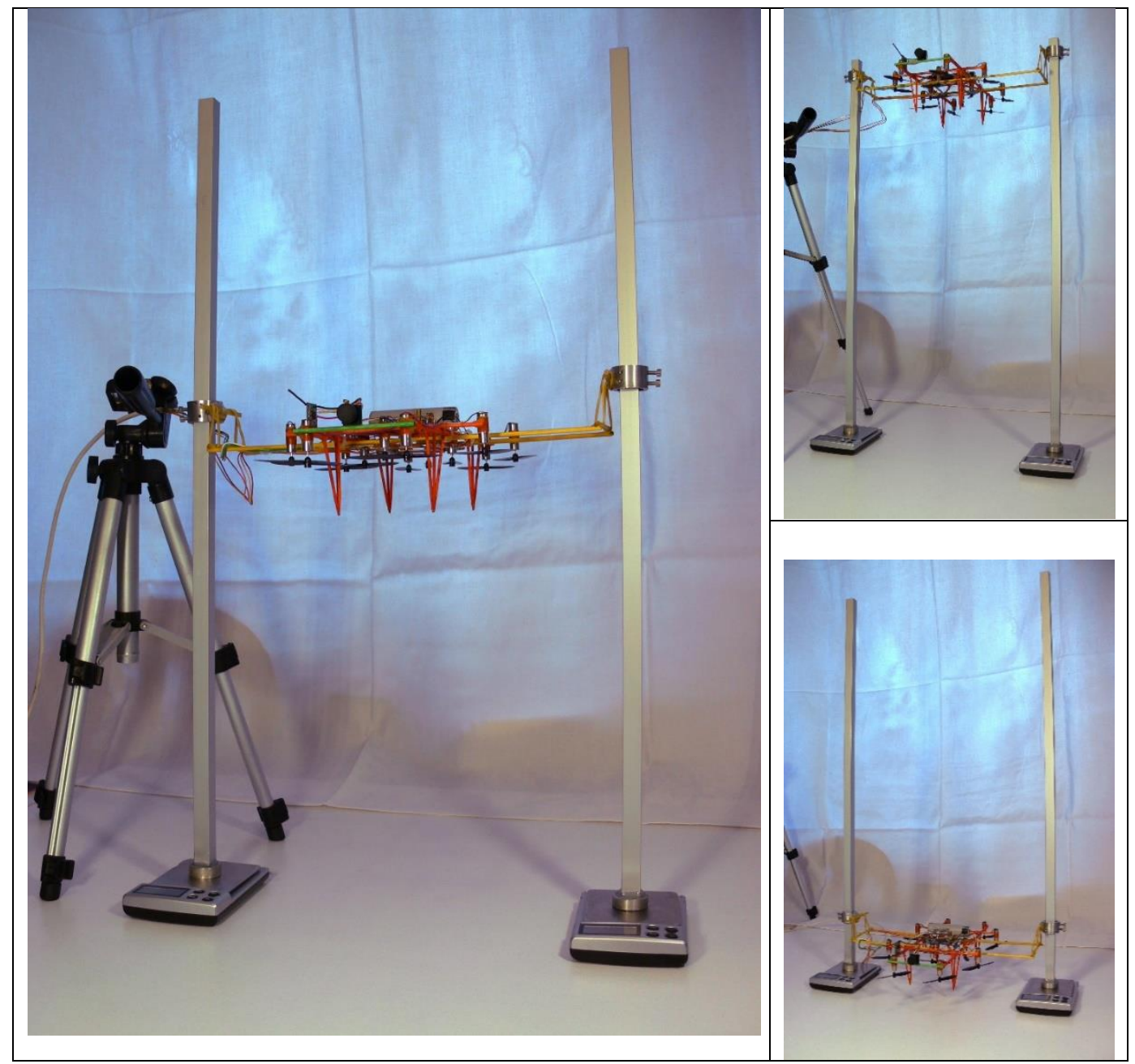

Figure 7 - Testing the undermount configuration for measuring lift to power ratio of the aircraft at constant power input. Measurements are taken from $4 \mathrm{~cm}$ to $50 \mathrm{~cm}$ distance of propeller discs to ground in $1 \mathrm{~cm}$ steps.

The benefits, which the motor scales test setup showed, are promising, but there are obviously certain obstacles in achieving such a large increase in lift to power ratio when this method is employed in the real prototype. The reasons are as follows:

- The scales arm has a much larger diameter than the fuselage's thin beams

- Part of the rotor induced flow is still passing through the beamstructured fuselage and around all parts of the aircraft such as avionics, battery, etc., though at much lower speed 
- There is rotor-rotor interference that is larger for undermount propellers in comparison to overmount propellers (Yoon et al., 2017).

We wanted to measure if these drawbacks would still be overwhelmed by the advantages of undermount propellers, as measured and reported by Theys et al. (2016), for a quadcopter. Therefore, we established the test setup shown on Figure 7 and Figure 8 . The aircraft is suspended by flexible tethers from two independent pylons. The latter are made of duralumin and the suspension points may be easily altered in height during the testing process. The pylons are fixed on two digital weighing scales respectively with measurement range of $0-1000 \mathrm{~g}$ and accuracy of $0.1 \mathrm{~g}$.
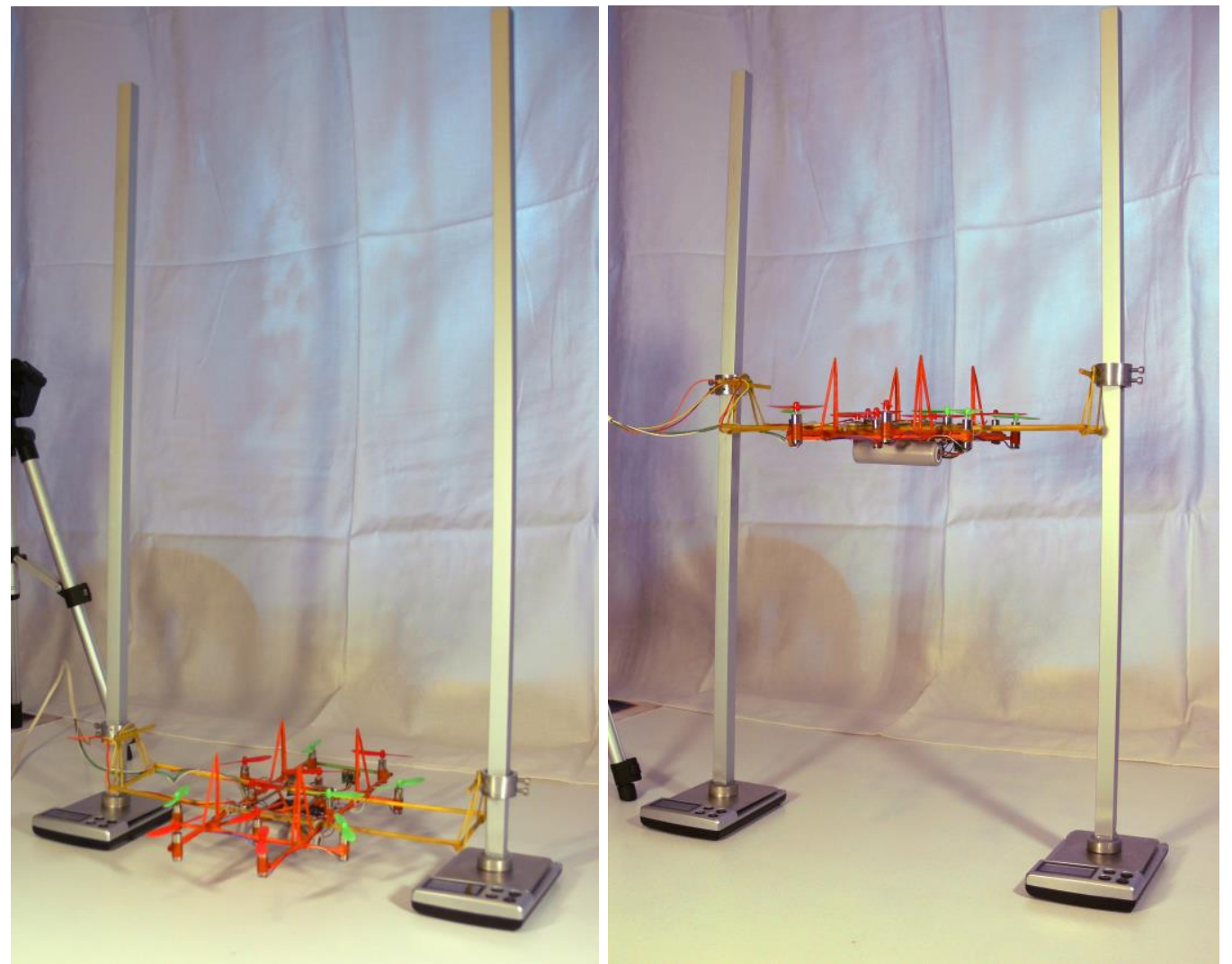

Figure 8 - Testing overmount propeller setup on the dodecacopter for measuring lift to power ratio. Again, the power input is constant and measurements are taken from $4 \mathrm{~cm}$ to $50 \mathrm{~cm}$ distance of propeller discs to ground in $1 \mathrm{~cm}$ steps.

The scales were pre-test calibrated. The prototype is further connected electrically to an external power supply with adjustable voltage control. The current and the voltage levels are monitored using digital voltmeter and ampere meter. The power 
cable is flexible and does not impede the measured lift. Prototype's battery and all avionics, including the autopilot are electrically disconnected. Once power is applied to the prototype, rotors start creating lift. The scales are nulled before each measurement and lift is directly measured on the scales as negative weights. Then the created lift is calculated as the sum of both scales sign-inverted indications. The generated lift is below the multi-rotor's weight. 


\begin{tabular}{|c|c|c|c|c|c|c|c|}
\hline \multirow[b]{2}{*}{$\begin{array}{l}\text { Height } \\
{[\mathrm{cm}]}\end{array}$} & \multicolumn{3}{|c|}{$\begin{array}{l}\text { Undermount } \\
\text { rotors }\end{array}$} & \multicolumn{3}{|c|}{$\begin{array}{l}\text { Overmount } \\
\text { rotors }\end{array}$} & \multirow[b]{2}{*}{$\begin{array}{l}\text { Lift to power } \\
\text { ratio gain: } \\
\mathrm{G}= \\
(\mathrm{LP} / \mathrm{LPo}- \\
1) * 100[\%]\end{array}$} \\
\hline & $\begin{array}{l}\text { Lift } \\
(\mathrm{Lu}) \\
{[\mathrm{g}]}\end{array}$ & $\begin{array}{l}\text { Lift } \\
{[\mathrm{N}]}\end{array}$ & $\begin{array}{l}\text { Lift to } \\
\text { power } \\
\text { ratio LPU } \\
{[\mathrm{g} / \mathrm{W}]}\end{array}$ & $\begin{array}{l}\text { Lift } \\
\text { (Lo) } \\
{[\mathrm{g}]}\end{array}$ & $\begin{array}{l}\text { Lift } \\
{[\mathrm{N}]}\end{array}$ & $\begin{array}{l}\text { Lift to } \\
\text { power } \\
\text { ratio LPo } \\
{[\mathrm{g} / \mathrm{W}]}\end{array}$ & \\
\hline 4.0 & 95.5 & 0.937 & 7.16 & 92.0 & 0.902 & 6.90 & 3.80 \\
\hline 5.0 & 94.2 & 0.924 & 7.06 & 90.4 & 0.887 & 6.78 & 4.20 \\
\hline 6.0 & 92.8 & 0.910 & 6.96 & 89.1 & 0.874 & 6.68 & 4.15 \\
\hline 7.0 & 91.9 & 0.901 & 6.89 & 87.9 & 0.862 & 6.59 & 4.55 \\
\hline 8.0 & 91.5 & 0.897 & 6.86 & 87.0 & 0.853 & 6.52 & 5.17 \\
\hline 9.0 & 91.3 & 0.895 & 6.84 & 86.4 & 0.847 & 6.48 & 5.67 \\
\hline 10.0 & 90.8 & 0.890 & 6.81 & 85.8 & 0.841 & 6.43 & 5.83 \\
\hline 11.0 & 90.7 & 0.889 & 6.80 & 85.5 & 0.838 & 6.41 & 6.08 \\
\hline 12.0 & 90.5 & 0.888 & 6.78 & 85.2 & 0.836 & 6.39 & 6.22 \\
\hline 13.0 & 90.3 & 0.886 & 6.77 & 84.7 & 0.831 & 6.35 & 6.61 \\
\hline 14.0 & 90.2 & 0.885 & 6.76 & 84.4 & 0.828 & 6.33 & 6.87 \\
\hline 15.0 & 89.8 & 0.881 & 6.73 & 84.2 & 0.826 & 6.31 & 6.65 \\
\hline 16.0 & 89.7 & 0.880 & 6.72 & 84.0 & 0.824 & 6.30 & 6.79 \\
\hline 17.0 & 89.5 & 0.878 & 6.71 & 83.9 & 0.823 & 6.29 & 6.67 \\
\hline 18.0 & 89.4 & 0.877 & 6.70 & 83.7 & 0.821 & 6.27 & 6.81 \\
\hline 19.0 & 89.5 & 0.878 & 6.71 & 83.5 & 0.819 & 6.26 & 7.19 \\
\hline 20.0 & 89.3 & 0.876 & 6.69 & 83.3 & 0.817 & 6.24 & 7.20 \\
\hline 21.0 & 89.1 & 0.874 & 6.68 & 83.4 & 0.818 & 6.25 & 6.83 \\
\hline 22.0 & 89.2 & 0.875 & 6.69 & 83.3 & 0.817 & 6.24 & 7.08 \\
\hline 23.0 & 89.4 & 0.877 & 6.70 & 83.4 & 0.818 & 6.25 & 7.19 \\
\hline 24.0 & 89.3 & 0.876 & 6.69 & 83.4 & 0.818 & 6.25 & 7.07 \\
\hline 25.0 & 89.2 & 0.875 & 6.69 & 83.4 & 0.818 & 6.25 & 6.95 \\
\hline 26.0 & 89.2 & 0.875 & 6.69 & 83.3 & 0.817 & 6.24 & 7.08 \\
\hline 27.0 & 89.4 & 0.877 & 6.70 & 83.3 & 0.817 & 6.24 & 7.32 \\
\hline 28.0 & 89.3 & 0.876 & 6.69 & 83.1 & 0.815 & 6.23 & 7.46 \\
\hline 29.0 & 89.1 & 0.874 & 6.68 & 83.2 & 0.816 & 6.24 & 7.09 \\
\hline 30.0 & 89.1 & 0.874 & 6.68 & 83.2 & 0.816 & 6.24 & 7.09 \\
\hline 31.0 & 89.2 & 0.875 & 6.69 & 83.1 & 0.815 & 6.23 & 7.34 \\
\hline 32.0 & 89.1 & 0.874 & 6.68 & 83.2 & 0.816 & 6.24 & 7.09 \\
\hline 33.0 & 89.3 & 0.876 & 6.69 & 83.4 & 0.818 & 6.25 & 7.07 \\
\hline 34.0 & 89.3 & 0.876 & 6.69 & 83.2 & 0.816 & 6.24 & 7.33 \\
\hline 35.0 & 89.2 & 0.875 & 6.69 & 83.3 & 0.817 & 6.24 & 7.08 \\
\hline
\end{tabular}




\begin{tabular}{|l|lll|lll|l|}
36.0 & 89.3 & 0.876 & 6.69 & 83.3 & 0.817 & 6.24 & 7.20 \\
37.0 & 89.1 & 0.874 & 6.68 & 83.2 & 0.816 & 6.24 & 7.09 \\
38.0 & 89.1 & 0.874 & 6.68 & 83.1 & 0.815 & 6.23 & 7.22 \\
39.0 & 89.0 & 0.873 & 6.67 & 83.3 & 0.817 & 6.24 & 6.84 \\
40.0 & 89.1 & 0.874 & 6.68 & 83.2 & 0.816 & 6.24 & 7.09 \\
41.0 & 89.3 & 0.876 & 6.69 & 83.1 & 0.815 & 6.23 & 7.46 \\
42.0 & 89.2 & 0.875 & 6.69 & 83.3 & 0.817 & 6.24 & 7.08 \\
43.0 & 89.1 & 0.874 & 6.68 & 83.4 & 0.818 & 6.25 & 6.83 \\
44.0 & 89.2 & 0.875 & 6.69 & 83.2 & 0.816 & 6.24 & 7.21 \\
45.0 & 89.2 & 0.875 & 6.69 & 83.3 & 0.817 & 6.24 & 7.08 \\
46.0 & 89.0 & 0.873 & 6.67 & 83.1 & 0.815 & 6.23 & 7.10 \\
47.0 & 89.3 & 0.876 & 6.69 & 83.0 & 0.814 & 6.22 & 7.59 \\
48.0 & 89.2 & 0.875 & 6.69 & 83.1 & 0.815 & 6.23 & 7.34 \\
49.0 & 89.0 & 0.873 & 6.67 & 83.2 & 0.816 & 6.24 & 6.97 \\
50.0 & 89.1 & 0.874 & 6.68 & 83.1 & 0.815 & 6.23 & 7.22 \\
\hline
\end{tabular}

Table 2. Test measurements

This test was intended to measure the efficiency increase in lift versus power, i.e. the increase of lift to power ratio of the aircraft when using undermount off-body propellers, instead of overmount rotors, but we broadened the experiment to also measure the ground effect of the multi-copter in both situation - with undermount and overmount rotors. Thus, we conducted the test starting at a very low distance of the rotors from ground $-4 \mathrm{~cm}$ - then proceeding to $50 \mathrm{~cm}$ where ground effect should have totally diminished. The step of measurement is $1 \mathrm{~cm}$. At each step the power delivered to the motors was adjusted to be the same (constant for the whole test) and five measurements at each height were performed and then averaged as the final result for that height.

First, undermount setup was tested using pusher propellers in black colour (Figure 7). After this test, all motors were refitted with tractor propellers (green and orange coloured propellers) and the prototype was turned upside down - see Figure 8.

The data from the two tests are shown in Table 2. A graphical representation of the data from Table 2 is presented on Figure 9. Table 2 has 47 rows starting from $4 \mathrm{~cm}$ rotor to ground distance and ending at $50 \mathrm{~cm}$ with $1 \mathrm{~cm}$ step. For the undermount setup there are three columns in the table, namely "Lift in grams for undermount rotors (Lu)," "The same lift but this time in Newtons," "Lift to power ratio in grams per Watt for the undermount setup (LPU)." The overmount setup has another three columns in Table 2 - "Lift in grams for overmount rotors (Lo)," "The same lift in Newtons," "Lift to power ratio in grams per Watt for the overmount setup (LPo)." 
The last column in Table 2 is the "Lift to power ratio gain in \% $(\mathrm{G})$ " and is calculated as follows:

$$
G=\left(\frac{L P_{U}}{L P_{O}}-1\right) * 100
$$

The tests were carried out at $13.34 \mathrm{~W}$ power delivered to the aircraft with all avionics including the autopilot disconnected.

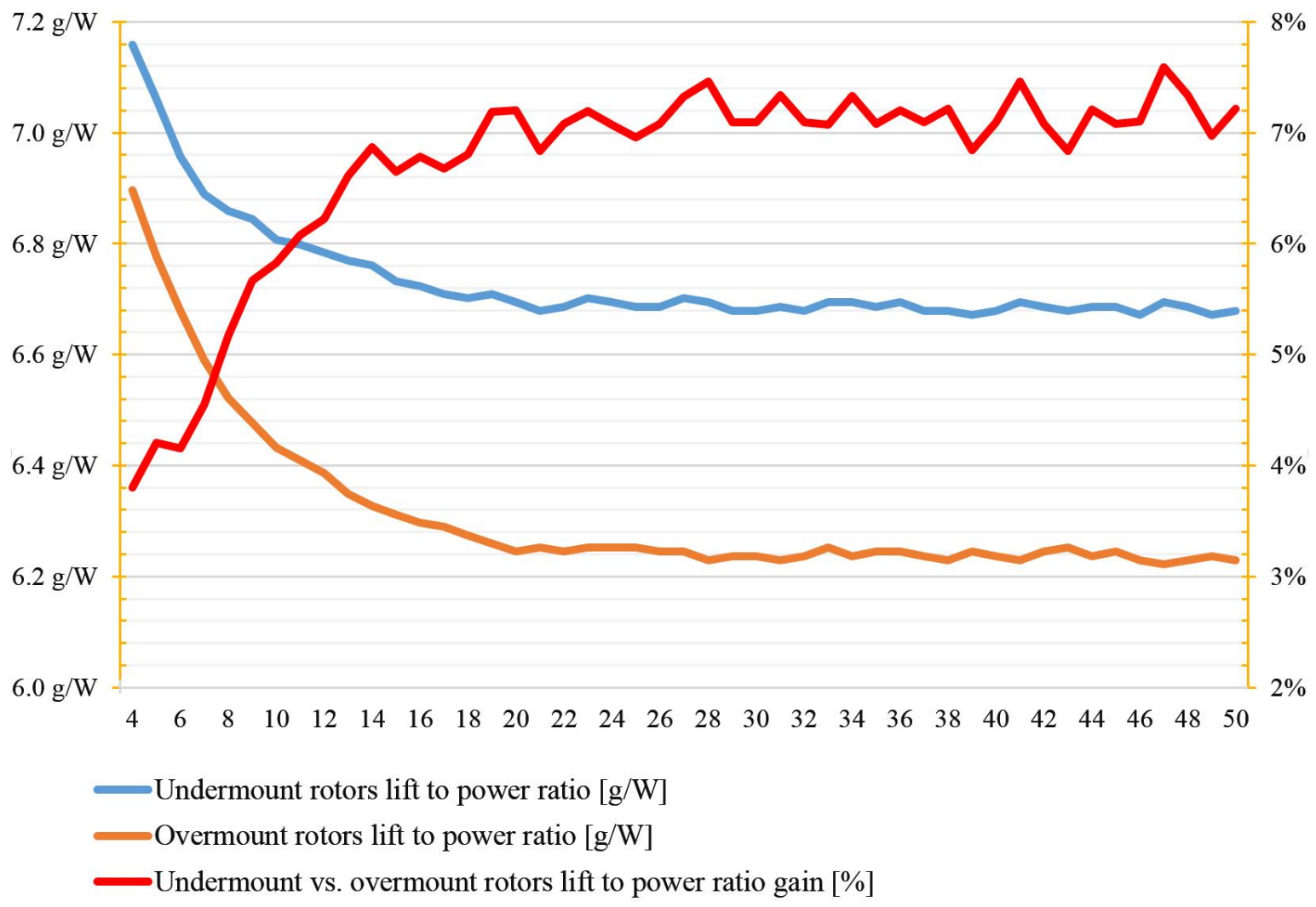

Figure 9 - Graphical presentation of the test results from Table 2. The g/W vertical axis to the left refers to the lift to power ratio measurements, while the percentage vertical axis to the right shows the lift to power ratio gain of using undermount instead of overmount rotors.

\section{Test results analysis and ground effect manifestation}

The results from Table 2 are graphically presented on Figure 9, where one could observe the line-charts of the measured lift to power ratios when using undermount (blue line) and overmount (orange line) propellers. The ratios are in 
grams per Watt. The gain in lift to power ratio is presented as a red line and is calculated according to formula (2) as already stated above. It is shown in \%. The gain is not as significant as with the rotor measuring scales test where it was $14.7 \%$ and this result was expected, but it is still significant and out of ground effect is stabilized around 7\%. This is a definite result supporting the undermount off-body scheme of employing rotors in the current dodecacopter design. This result is also in agreement with other experiments (Theys et al., 2016).

Further, the ground effect was studied and one may observe its manifestation in the graphic chart in Figure 9 (or by reading Table 2). The ground effect in both undermount and overmount configurations starts at approximately $18 \mathrm{~cm}$ height of the rotors from ground. With a rotor radius of $5.5 \mathrm{~cm}$ we obtain a height of 6.5 rotor radii where the ground effect starts to be visible. This value is higher than the well-established one for single rotor helicopters, which is around 2 rotor radii (Cheeseman \& Bennett, 1955; Fradenburgh, 1960). For quadcopters, Sanchez-Cuevas, Heredia, and Ollero (2017) measured the same height to be around 5 rotor radii.

\section{Conclusions}

The benefits obtained from the model of the Bulgarian Knight multi-copter have motivated the authors to continue their work on other models of multi-rotors implementing the same paradigms - undermount pusher propellers and honeycomb packing of the rotors.

A conclusion may be drawn that increasing the rotor number from 1 to 12 also increases the upper bound of ground effect manifestation from 2 to 6.5 rotor radii.

To test the conceptual models, the authors choose to develop micro-drone prototypes weighing less than $250 \mathrm{~g}$, because of the currently increased interest in such aircraft, determined by their unique capabilities of flying indoors, passing through windows, being silent and hard to spot visually, as well as by radar.

The suggested design in this paper is patent pending at the Bulgarian Patent Office and has been awarded a gold medal from Tesla Fest 2016 International Festival of Innovations, Knowledge and Creations, Novi Sad, Serbia. 


\section{References}

Block, P. J. W. (1986). Experimental study of the effects of installation on singleand counter-rotating propeller noise. Retrieved from https://ntrs.nasa.gov/ archive/nasa/casi.ntrs.nasa.gov/19860016690.pdf

Cheeseman, I., \& Bennett, W. (1955), The effect of the ground on a helicopter rotor in forward flight. ARC R\&M 3021. Retrieved from http://naca.central.cranfield.ac.uk/reports/arc/rm/3021.pdf

Fradenburgh, E. A. (1960), The helicopter and the ground effect machine, Journal of the American Helicopter Society, 5(4), 24-33.

Hanson, D. B. (1985), Noise of counter-rotation propellers, Journal of Aircraft, 22(7), 609-617.

Maisel, M. D., Giulianetti, D. J., \& Dugan, D. C. (2000). The history of the XV15 tilt rotor research aircraft: From concept to flight. NASA SP-2000-4517. Retrieved from https://history.nasa.gov/monograph17.pdf

Marichal, G. N., Rodriguez, M. T. \& Rivera, S. C. (2013). Vibration reduction for vision systems on board unmanned aerial vehicles using a neuro-fuzzy controller, Journal of Vibration and Control. doi:10.1177/1077546313479632

Piercy, J. E., \& Embleton, T. F. W. (1977). Review of noise propagation in the atmosphere. The Journal of the Acoustical Society of America, 61, 1403.

Radkowski, S. \& Szulim, P. (2014). Analysis of vibration of rotors in unmanned aircraft. 2014 19th International Conference on Methods and Models in Automation and Robotics (MMAR).

Sanchez-Cuevas, P., Heredia, G. \& Ollero, A. (2017). Characterization of the aerodynamic ground effect and its influence in multirotor control. International Journal of Aerospace Engineering . doi:10.1155/2017/1823056

Steinhaus, H. (1999), Mathematical snapshots (3rd ed). New York: Dover.

Theys, B., Dimitriadis, G., Hendrick, P. \& DeSchutter, J. (2016). Influence of propeller configuration on propulsion system efficiency of multi-rotor Unmanned Aerial Vehicles. 2016 International Conference on Unmanned Aircraft Systems (ICUAS).

Verbeke, J., \& Debruyne, S. (2016, September), Vibration analysis of a UAV multirotor frame. International Conference on Noise and Vibration Engineering.

Yoon, S., Diaz, P. V., D., Boyd, D. Jr., Chan, W. M., \& Theodore, C. R. (2017, May 8). Computational aerodynamic modeling of small quadcopter vehicles. 73rd Annual AHS International Forum and Technology Display 2017 (AHS Forum 73). 